\title{
Improving access to multilingual health information for newcomers to Canada ${ }^{1}$
}

\author{
Valeria Gallo Stampino
}

\begin{abstract}
Canada's immigrant and refugee population is a vulnerable group in our health care system with specific information needs. Newcomers to Canada face certain socioeconomic, cultural-linguistic, and systemic barriers to access to health care that government, social agencies, and health care organizations work to overcome. To address some of the communication barriers, many health organizations develop information resources such as online brochures and education handouts. Several organizations offer specifically tailored multilingual publications to meet newcomers' information needs and write them using cross-cultural approaches. However, multilingual health information may be hard to locate and is not readily available through major Canadian consumer Web sites. This article discusses the advantages of sharing multilingual publications online and asks whether a central portal or repository is a possible solution for making publications more widely available across Canada.
\end{abstract}

\section{Introduction}

This paper discusses the importance of providing access to health information for Canadian immigrants. Multilingual resources help newcomers overcome a number of barriers to understanding and navigating Canada's health care system. To improve and facilitate communication for vulnerable populations, many health organizations develop information resources in other languages and make them available online. This article discusses the advantages of sharing multilingual publications online and asks whether a central portal or repository is a possible solution for making publications more widely available across Canada.

\section{Background}

The number of immigrants and refugees in Canada reached 5.4 million in 2001, constituting $18.4 \%$ of the total population [1]. Some recent research suggests that new immigrants are among the most vulnerable of health populations; while their health status is good initially, their health tends to worsen during their first 10 years in Canada [2].

Though traditionally a nation of immigrants, Canada's historic patterns of immigration are changing, particularly, the countries of origin. While $62 \%$ of immigrants are currently of European origin, $74 \%$ of those who came to Canada in the last 10 years are from non-English speaking

V. Gallo Stampino. UBC School of Library, Archival and Information Studies, University of British Columbia,

6190 Agronomy Road, Vancouver, BC V6T 1Z3, Canada

(e-mail: valeriag@interchange.ubc.ca).

${ }^{1}$ This article was originally written as a requirement for a course on Health Information Sources and Services at the University of British Columbia's School of Library, Archival and Information Studies.
Asian, Latin American, and African countries [3]. The different composition of today's immigrants has an impact on access to care, creating specific problems for provision of information services.

\section{Immigrant access to health care}

In a recent Multicultural Health Fair in British Columbia, Sasso and Stanger [4] stated that immigrants face three main types of health barriers: socioeconomic, cultural-linguistic, and systemic. In terms of socioeconomic barriers, income disparities are among the major determinants of access to care [3]. Many newcomers believe that contact with health care providers will jeopardize their immigration status [5]. Immigrants face other obstacles that adversely affect their health and well-being; they suffer from abrupt changes in their everyday lives, with the loss of family ties and social networks [6]. Many would agree that "despite the practical, cultural and economic benefits that immigrants bring to Canada, there is a persistent undercurrent of discrimination and racism among many established Canadians and among many incoming immigrant groups" [7].

Of the 424000 new immigrants that came to British Columbia (B.C.) between 1992 and 2001, 50\% did not speak, read, or write English [4]. Often, children in the family translate for health care professionals and family members, thereby creating other barriers. Low literacy levels have a direct and indirect impact on the health of Canadians [8]. Cultural differences raise barriers as health care systems in other countries are substantially different from those in Canada. Newcomers bring their values, beliefs, and a set of expectations about how to interact with health care practitioners, which may conflict with Canadian values [6].

Health, social services, and government bureaucracy are identified as systemic problems because immigrants find them difficult to understand. In fact, "many people spend 
scarce time and money going from one office to another to obtain clarification of status and medical insurance" [6]. Access to information that supports programs and services is also a challenge. Many agree that the responsibility for access to the system and communication among cultures rests on a system that is poorly designed to accommodate newcomers' needs [4].

\section{Interventions}

In Canada, a number of initiatives aim to ensure that nonnative English speakers can improve their communication with the health care system. Many include a cross-cultural approach and recognize the need to tailor health promotion initiatives to specific immigrant subgroups. The need to acknowledge ethnographic characteristics has caused a shift of perspective in health, considered by many as "a social process in which each party - the professional and the patient brings a set of beliefs, expectations, and practices to the encounter" [6]. Cross-cultural approaches to health recognize different cultures of medicine and send the message that no system is better or more valid than others. This approach to health care conforms to the Multicultural Policy of Canada of 1971 [9].

The health promotion literature suggests that "tailoring" health initiatives to individual populations is better than "targeting" them with general announcements. Tailoring offers customization and includes health messages and resources that conform to the needs and cultural beliefs of a subpopulation rather than simply trying to expose that subgroup to standard messages [10]. For example, the North Hamilton Community Health Centre in Ontario started its Immigrant/Refugee Health Programme in 1989 to "overcome the language, cultural and information services barriers that prevent new immigrants and refugees from using health and social services" [3]. The initiative includes medical staff that speak other languages and offer workshops on different health topics. Such integrated programs are available in many places across Canada.

Foreign-language interpreters are available in some jurisdictions in Canada. British Columbia offers translation services for various linguistic groups; a toll-free multilingual health information line is available, for example. Many nurses are trained in basic communication skills in other languages or rely on various techniques to communicate, such as the use of translation cards. A local example is Vancouver's Cross Cultural Mental Health Services which aims to "develop health care that acknowledges racial and cultural diversities" [9]. Among the steps taken to meet this goal is matching a patient's cultural and language needs with appropriate staff, offering orientation sessions for the public, and training staff in other languages. Public education is emphasized by the creation of health pamphlets in the major languages spoken in the community, as well as offering information in other languages via the organization's Web site.

As a result of cross-cultural care training, health information materials are either specifically designed or carefully translated and adapted for newcomers. When health professionals provide information resources in other languages, they choose culturally sensitive materials in favour of general information available on the Web.

\section{Providing access to multilingual health resources}

Many issues that Canadian immigrants and refugees deal with come from a lack of information, and several strategies are emerging to address these needs. Information and educational materials developed in hospitals and organizations are useful, not to mention those developed for community organizations, health clinics, and settlement agencies. In addition to covering basic health, the resources provide information on how to navigate the health system and what support is available to assist newcomers.

Multilingual health education materials are usually provided when immigrants contact immigration and health care agencies. Materials are available in public places such as libraries or community centres and online as traditional text-based resources or in multimedia formats. In Ontario, the Ministry of Health and Long-Term Care's Web site (http://www.health. gov.on.ca/english/public/program/hepc/hepc_factsheets.html) includes resources in Arabic, Punjabi, Italian, Hindi, Vietnamese, and Filipino. In B.C., the B.C. Health Guide Web site (http://www.bchealthguide.org/multicultural.stm) provides information on health topics in French, Chinese, Punjabi, and Farsi.

Web-accessible publications help to simplify sharing for agencies that specialize in health information materials and services for immigrants. Sharing resources among agencies increases the likelihood that they will be accessed by newcomers. In this sense, public librarians may be better positioned to reach people who avoid medical and social services. As opposed to print, which may be limited in numbers, online resources are available to anyone with an Internet connection. Even though not all users will have direct access to online resources, health workers and other professionals can print and hand them to newcomers as needed. For these reasons, many organizations keep copies of the resources they produce in electronic format.

Providing access to multilingual resources created by various health organizations is a challenge for information professionals.

A variety of health topics in many languages are available, but some materials are geared to a limited audience. Today's frequent institutional changes in health care affect resource collections that are either moved from one location to another or other times disappear [11]. In some cases, patient care resources are accessible on hospital Intranets or internal Web pages only and are not searchable via search engines. Bringing resources together in one place might improve access to and use of these resources by Canadian health organizations.

The difficulties of access to multilingual materials prevent some health care agencies from easily identifying available topics and languages, and topics that need to be developed. Health information professionals can contribute to organizing, developing, and improving access to these resources. Information professionals often mediate between local settlement 
agencies, community organizations, health care professionals, government agencies, and the general public regarding the particular information needs of different communities. It makes sense to share resources and avoid duplication wherever possible, and health librarians should ensure that this occurs.

\section{The big picture}

As of early 2007, Canadian health librarians do not have a central Canadian repository for multilingual resources. None of the "top 10" Canadian consumer health Web sites [12] on a list compiled by the Consumer Health Information Providers Group have sufficient multilingual coverage. While Web sites such as the Canadian Medical Association public Web site (http://www.cma.ca/public) provide links to useful health information, materials are available in English or French only. Materials at the Canada Health Portal (http://www.healthportal.gc.ca) are also written in English and French, with some coverage in other languages. Health Canada's Web site (http://www.hc-sc.gc.ca) lacks entry points for recent immigrants who speak other languages. The Canadian Health Network (http://www.canadian-health-network.ca) includes materials in other languages, but it does not provide coherent organization or searching. That said, its links to organizations with resources in other languages are useful.

A review of major Canadian consumer health Web sites suggests that more multilingual health resources need to be developed. Common categories for health information by population on these national Web sites are frequently limited to children, youth, seniors, Aboriginal Peoples, women, and men. Some health information is available for immigrants in their mother tongue but is hard to locate. Given the number of immigrants coming to Canada each year, and a need to provide health information in other languages, this information should be more widely offered.

\section{Conclusion}

Whether more multilingual resources can be added to the major Canadian consumer health Web sites in the future remains to be seen. More cooperation and sharing of resources should continue at municipal and provincial levels, given the characteristics of the Canadian health system and Canada's shifting demographics.

Curiously, many of the obstacles involved in establishing networks of multilingual health resources for newcomers are similar to those identified by the Canadian Health Libraries Association / Association des bibliothèques de la santé du Canada (CHLA / ABSC) National Network of Libraries of Health taskforce. Among those obstacles are the crossjurisdictional nature of the Canadian health care system and the cost of establishing a body to coordinate national initiatives [13], for example.

Multilingual health resources should be added to the major Canadian health portals for a number of reasons. First, new immigrants should be able to access them to learn about Canada's health care system and the different responsibilities for providing access to health care services among levels of government. Second, improvements to access would assist health librarians and health professionals by collating the many reputable Canadian sources in one location. By allowing the recognition of substantial topical or language gaps, a central portal would avoid the duplication of patient education materials in other languages. Many benefits exist in the sharing of information across health centers, settlement agencies and government agencies.

In conclusion, immigrants cope with many difficulties in terms of access to health care and obtaining information to understand the health care system. Multilingual resources help newcomers navigate the health system and address communication barriers. Though many organizations have developed useful multilingual resources, difficulties exist in accessing them. The advantages of sharing these resources digitally are obvious: avoiding duplication, identifying gaps, and reaching new users. The lack of multilingual content in Canadian health portals and the desirability of adding content require further attention by health librarians; further cooperation across organizations and sectors should be explored. Health library and information professionals via CHLA / ABSC might be a useful starting point in leading any future initiatives, especially if the goal is to provide more coherent access to multilingual health information resources in Canada.

\section{References}

1. Ng E, Wilkins R, Gendron F, Berthelot JM. Dynamics of immigrants' health in Canada: evidence from the $\mathrm{Na}$ tional Population Health Survey [cited 2006 Nov 20]. Available from http://www.statcan.ca/english/research/82-618MIE/2005002/pdf/82-618-MIE2005002.pdf.

2. Canadian Institutes of Health Research. Interdisciplinary capacity enhancement grants - Reducing health disparities and promoting equity for vulnerable populations [homepage on the Internet]. 2005 [cited 2006 Nov 20]. Available from http://www.cihr-irsc.gc.ca/e/25703.html.

3. Fowler N. Providing primary health care to immigrants and refugees: the North Hamilton experience. CMAJ. 1998;159:388-91. Available from http://www.pubmedcentral.nih.gov/articlerender. fcgi?artid=1229607.

4. Sasso A, Stanger E. Accessible health care: a response framework for a culturally evolving community [monograph on the Internet]. Vancouver: Affiliation of Multicultural Societies and Service Agencies of B.C. (AMSSA); 2005 [cited 2006 Nov 20]. Available from http://www.amssa.org/multiculturalhealthyliving/phase1/images/ resources/healthbriefing_final.pdf.

5. Palacios C, Sheps S. A pilot study assessing the health status of the Hispanic American community living in Vancouver. Can J Public Health [serial on the Internet]. 1992 [cited 2006 October 31]; 83(5):346-9. Available from CINAHL with Full Text.

6. Waxler-Morrison N, Anderson JM, Richardson E, editors. Crosscultural caring: a handbook for health professionals. Vancouver: UBC Press; 1990.

7. Caufield C. Health of immigrant Hispanic families: narrative and phenomenological hermeneutics. Nurse Res. 2006;13(3):22-31.

8. Rootman I, Ronson B. Statistics Canada. Literacy and health research in Canada: where have we been and where should we go? Can J Public Health. 2005;96(2). Available from http://www.igh.ualberta.ca/RHD/Synthesis/Literacy.htm. 
9. Ganesan S, Janz T. Overview of culturally-based mental health care in Vancouver. Transcult Psychiatry. 2005;42(3):478-90. Available from http://tps.sagepub.com/cgi/content/abstract/42/3/478.

10. Hyman I, Guruge S. A review of theory and health promotion strategies for new immigrant women. Can J Public Health. 2002 May/Jun;93(3):183-7.

11. Marton C. Environmental scan on women's health information resources in Ontario, Canada. Information Research. 2001 [cited 2006 Nov 20; updated 2001 Sept 26]; 7(1). Available from http://InformationR.net/ir/7-1/paper116.html.

12. Murray S. Consumer health information. JCHLA [serial on the Internet]. 2006 [cited 2007 Feb 23]; 27(3):75-6. Available from http://pubs.nrc-cnrc.gc.ca/jchla/jchla27/c06-026.pdf.

13. McGowan J, Straus SE, Tugwell P. Canada urgently needs a national network of libraries to access evidence. HealthcQ. 2006;9(1):72-4. 